\title{
Managing post-traumatic trigeminal neuropathic pain: is surgery enough?
}

\author{
John R. Zuniga ${ }^{1 *}$, Tara F. Renton ${ }^{2}$
}

'Departments of Surgery and Neurology and Neurotherapeutics, The University of Texas Southwestern Medical Center at Dallas, Dallas, Texas, USA ${ }^{2}$ Department of Oral Surgery, Kings College London Dental Institute, Denmark Hill Campus, London SE5 9RS, UK

\section{Article Info}

\section{Article Notes}

Received: September 19, 2016

Accepted: October 08, 2016

\section{${ }^{*}$ Correspondence:}

John R. Zuniga DMD, MS, PhD, Departments of Surgery and Neurology and Neurotherapeutics, The University of Texas Southwestern Medical Center at Dallas, Dallas, Texas, USA, Email: John.Zuniga@UTSouthwestern.edu

(c) 2016 Zuniga JR. This article is distributed under the terms of the Creative Commons Attribution 4.0 International License

\section{Keywords:}

Trigeminal Nerve

Neuropathic Pain

Trigeminal Nerve Microsurgery

\section{ABSTRACT}

In the absence of effective non-surgical methods to permanently resolve neuropathic pain involving the lip, chin, or tongue following inferior alveolar and/or lingual nerve injury, microsurgery of these nerves has been a recommended modality. In two ambispective clinical trials, we demonstrated that phenotypic differences exist between individuals with neuropathic pain and those without neuropathic pain of the trigeminal nerve. In those without neuropathic pain before microsurgery there was a $2 \%$ incidence of neuropathic pain after microsurgery whereas there was a $67 \%$ incidence of neuropathic pain after microsurgery, some reporting an increase in pain levels, when neuropathic pain was present before microsurgery. The recurrence of neuropathic pain after trigeminal microsurgery is likely multifactorial and might not depend on factors that normally affect useful or functional sensory recovery in those who have no neuropathic pain. These results indicate that the understanding of post-traumatic trigeminal neuropathic pain is incomplete. Predictive outcomes of treatment will probably improve when the etiology is better defined to allow mechanistic or target-/site-specific treatment. Until then, non-surgical treatment for post-traumatic trigeminal neuropathic pain remains a safer option. Risk factors have been identified for patients developing chronic post -surgical pain due to post-traumatic neuropathy. These include psychological, medical, and age related factors. The best management may lie in preoperative screening and avoidance of elective surgery for high risk patients as the prevention of post-traumatic trigeminal neuropathic pain in the absence of effective medical or surgical interventions.

\section{Introduction}

The intuitive premise that a peripheral nerve injury should be resolved with a peripheral nerve procedure is challenged when neuropathic pain is a component condition. Modern microneurosurgeons espouse that motor, sensory and mixed functional recovery in crushed, partially transected and completely transected peripheral nerves occur when proper techniques are employed to reconnect healthy proximal and distal nerve endings dependent, in part, upon age, duration, location and type of nerve injury. Neuropathic pain can occur as a result of peripheral nerve injury and presents as allodynia, hyperpathia, hyperalgesia, and sympathetic mediated characteristic pain reactivity in and around the dermatome affected when a sensory or mixed nerve is injured. The inferior alveolar nerve (IAN) carries general sensation for the mouth, teeth, lip and chin and the lingual nerve (LN) carries general and special sensation (taste) for the anterior two-thirds of the tongue, floor of mouth and lingual mucosa of the mouth. Both 
are branches of the third division of the trigeminal nerve and serve important function for oral health and general functions such as eating, chewing, tasting, and phonation.

The incidence of injury to either of these nerve by elective dental or oral and maxillofacial surgical procedures ranges from $0.6 \%$ to $90 \%{ }^{1,2,3}$. The most common cause of injury, in order, is third molar extraction, reconstructive mandibular surgery, mandibular trauma, dental injection, dental implant placement, dental endodontic therapy, or the consequence of pathology of the oral cavity or surgery for pathology of the oral cavity ${ }^{4,5}$. The most common outcome of nerve injury is the loss of general sense or general and special sense of the lip/chin/tongue depending upon which nerve is injured (IAN provides sensation to the lip/chin and LN the tongue). The loss of general sensation of the lip and chin can be measured by clinical testing ${ }^{6}$ and the degree of injury can be visualized by special imaging, magnetic resonance neurography ${ }^{7}$. The loss of general sensation and taste capability of the anterior tongue can also be measured by clinical testing and videography ${ }^{6,8}$ and the degree of injury can be visualized by special imaging, magnetic resonance neurography ${ }^{7}$. The majority of injured IAN and LN recover spontaneously but spontaneous recovery depends upon the modality and the severity of the injury (compression, partial or complete transection) that would or would not support neurotization of injured axons $^{9,10,11,12,13,14,15,16}$. Based on time from injury, degree of nerve injury, location and age of the patient, surgical reconnection of the injured nerve or decompression results in functional or useful sensory recovery in general sensation or general/special sensation in up to $90 \%$ of patients.

\section{Clinical presentation}

The patient with iatrogenic post-traumatic trigeminal neuropathy may present with anesthesia, hypoesthesia or hyperesthesia, all with attendant functional problems and resultant psychological sequelae. The issues specific to trigeminal pain include the complexity of the region, the problematic impact on daily function and significant psychological impact ${ }^{17}$. By nature of the geography of the pain (affecting the face, eyes, scalp, nose, mouth) it may interfere with just about every social function we take for granted and enjoy ${ }^{18}$. The trigeminal nerve is the largest sensory nerve in the head and neck, protecting the essential organs that underpin our very existence (brain, eyes, nose, and mouth). It is no wonder that pain within the trigeminal system in the face is often overwhelming and inescapable for the affected individual.

of the injured trigeminal nerves, the incidence of developing post-traumatic trigeminal neuropathic pain has been reported to range from $0.45 \%$ to $70 \%{ }^{19,20}$ of injuries involving the IAN and LN. Clinical presentation of post-traumatic trigeminal neuropathy is similar to other sensory nerves; however, due to the anatomical location the functional and psychological impact is greater than most other nerve injuries ${ }^{21}$. Most studies purely report mechanosensory presentation; however, a more holistic approach for assessing the patients with trigeminal nerve injury is recommended ${ }^{18}$. There are 3 features of posttraumatic trigeminal neuropathy worthy of assessment. They are pain discomfort, functional implications, and psychological abnormalities ${ }^{18,22}$. Neuropathic pain is commonly experienced by $50-70 \%$ of patients as either spontaneous ongoing pain, which is often a burning characteristic, a spontaneous shooting, or electric shocklike sensation (neuralgia). Patients also experience stimulus induced pain due touch or cold often having difficulties with daily function, such as kissing, socializing, speech, eating and drinking. Consequently, patients were often anxious, tearful and had psychological repercussions and demonstrated personality trait disorder, anxiety, stress, post-traumatic stress disorder, anger, etc.

\section{Phenotypic differences}

Injury of the IAN and LN are common consequences for loss of general and general/special sense but also the base of the presentation of neuropathic pain in these nerve distributions. Since microsurgery of the IAN and LN is inherently an intentional injury of the same nerves, then one question that we asked was whether patients who did not have neuropathic pain before microsurgery developed neuropathic pain after microsurgery. Sixty - five patients who underwent microsurgery of the IAN $(n=25)$ and $\mathrm{LN}(\mathrm{n}=40)$ were evaluated in an ambispective study and recorded the presence $(n=17)$ or absence $(n=48)$ of neuropathic pain before and 3, 6, and 12 months after microsurgery. Only one patient of 48 (2\%) who had no neuropathic pain before microsurgery had developed neuropathic pain after microsurgery whereas 11 of 17 $(67 \%)$ who had neuropathic pain before surgery had pain after surgery. Neuropathic pain prior to microsurgery as a predictor of pain after microsurgery had a sensitivity of $91 \%$, specificity of $88 \%$, positive predictive value of $67 \%$ and a negative predictive value of $97 \%$. Thus, the presence of neuropathic pain prior to trigeminal microsurgery is the major risk factor for the continuation or recurrence of post-traumatic trigeminal neuropathic pain. Patients who had neuropathic pain before surgery were: (a) Older $(\mathrm{p}=0.02)$; (b) more likely to have an IAN injury rather than a LN injury ( $\mathrm{p}=0.003)$; (c) more likely to have had a greater duration of time from injury to repair $(p=0.04)$, and, (d) more likely to have a compression injury than a complete transection injury of the involved nerve $(p=0.01)^{23}$.

\section{Phenotypic difference in microsurgery response}

Although the majority of IAN and LN repairs in 
patients with post-traumatic trigeminal neuropathic pain before surgery had neuropathic pain after surgery, there were some whose pain was resolved or diminished. In another ambispective study of twenty-eight patients with neuropathic pain before surgery, the phenotypic profiles of patients who demonstrated complete resolution $(n=7)$, incomplete resolution $(\mathrm{n}=11)$, or complete recurrence/ progression $(n=10)$ of neuropathic pain was evaluated 3 , 6 , and 12 months postoperatively. Sixty-four percent or chance incidence for relief or reduction in neuropathic pain occurs with microsurgery of either the IAN or LN. There was no effect of age or gender, location of injury, degree of injury, cause of injury, duration of injury, type of repair, or health comorbidities within or between these groups $^{24}$.

\section{Trigeminal neuropathic pain}

Our understanding of the neuromechanism(s) that cause post-traumatic trigeminal neuropathic pain of the IAN and LN are still unknown. These findings only point out that significant phenotypic differences exist between individuals who have neuropathic pain versus those who do not after trigeminal nerve injuries. Age at the time of injury, the duration of time from injury to repair, the degree of nerve injury, and location/subtype of nerve (IAN $>\mathrm{LN}$ ) may predict onset.

Treatment includes microsurgery for trigeminal injuries as it does for non-trigeminal nerve injuries. However, at least for trigeminal, there does not appear to be a predictable benefit of surgery for neuropathic pain relief that exists for patients without neuropathic pain. There is no phenotypic marker(s) that predicts relief or reduction. We can only assume based on these ambispective clinical trials that until there is a recognized neuromechanism(s) for the onset of neuropathic pain in the sensory system will there be a reliable base for surgical intervention or a surgical intervention that is site specific and effective. Until then, microsurgery has only chance benefit.

\section{Management}

Generally a holistic approach is required based on pain, functional and psychological problems that the patient is suffering from. The iatrogenic nature of these injuries and their subsequent chronic pain lays a heavy psychological burden at the patient's door. Hence the need for psychological interventions through the patient's post-operative journey is essential, and evidence base is emerging ${ }^{21}$. The surgical and medical management will depend on the mechanism (Table 1) and the duration of the nerve injury and the patient's complaints. There may be limited window to maximize IAN injury resolution in relation to dental implants, endodontics, and third molar induced injuries. A report suggested that early removal of implant (within 30 hours) may maximize neuropathy resolution; however, the evidence remains weak $^{25}$. The pharmacologic therapies for acute trigeminal injuries include the use of corticosteroids and non-steroidal anti-inflammatories ${ }^{26,}{ }^{27}$. Late phase pharmacologic management includes using topical local anesthetics, anticonvulsants for stimulus or spontaneous pain, tricyclic antidepressants or serotonin reuptake inhibitors for constant or elicited burning pain ${ }^{28,29}$. Only $15 \%$ of patients use long term systemic medications for post-traumatic trigeminal neuropathic pain due to side effects and $18 \%$ of IAN injured patients use topical medications long term ${ }^{30}$. Botulinum toxin injections for focal neuropathic pain have limited success and facial palsy is a poorly tolerated side effect $^{31}$. Alternative pain management strategies are also proving effective for trigeminal neuropathic pain including TENS, acupuncture, and low level laser therapies ${ }^{32,33,34}$.

\section{Summary}

Clinical trials of post-traumatic trigeminal neuropathic pain have pointed out that phenotypic differences exist between individuals who have neuropathic pain before microsurgery of the damaged trigeminal nerve versus those who do not. Microsurgery has a predictable benefit

\begin{tabular}{|c|c|}
\hline EVENT & \\
\hline Any event & suspected or known nerve damage or section requires IMMEDIATE referral for repair \\
\hline Implant & $\begin{array}{l}<24-48 \text { hours surgery if neuropathy persists after LA has worn off consider removal of } \\
\text { implant } \\
\text { Do NOT backup, apicect or replace with shorter implant }\end{array}$ \\
\hline Endo & $\begin{array}{l}<24-48 \text { after neuropathy arises consider } \\
\text { - } \quad \text { surgery remove tooth, surgery to remove overfill or } \\
\text { instrumentation or } \\
\text { - } \quad \text { retain tooth and decompress IDC and irrigate with saline for hours }\end{array}$ \\
\hline Wisdom teeth- Inferior alveolar nerve injury & $\begin{array}{l}<2 \text { weeks surgery can be indicated with evidence of retained tooth fragments or damaged } \\
\text { IDC }\end{array}$ \\
\hline Wisdom teeth -Lingual Nerve Injury & > 3-6months surgery \\
\hline Local anaesthetic nerve injuries (LN or IAN) & Therapeutic management only \\
\hline Orthognathic nerve injuries & Therapeutic management only \\
\hline Mandibular fracture nerve injuries & Therapeutic management only \\
\hline
\end{tabular}

Table 1: Management for Post-Traumatic Trigeminal Neuropathy. 
for improving general sense and special sense in the trigeminal nerves but has only chance effect on neuropathic pain. Until actual neuromechanics or target-/site-specific causing neuropathic pain are identified, peripheral microneurosurgical intervention of the trigeminal nerves is questionable treatment.

A recent Cochrane Systematic review of the interventions for post-traumatic trigeminal neuropathy due to dental surgery concluded that there is clearly a need for randomized controlled clinical trials to determine the effectiveness of surgical, medical, and psychological interventions for iatrogenic IAN and LN injuries. Primary outcomes should include: patient-focused morbidity measures including, altered sensation and pain, quantitative sensory testing, the functional and psychological effects and effects of delayed treatment ${ }^{35}$.

\section{References}

1. Hillerup S. Iatrogenic injury to the inferior alveolar nerve: etiology, signs and symptoms, and observation on recovery. Int J Oral Maxillofac Surg. 2008;17:704-709.

2. Robert RC, Bacchetti P, Pogrel MA. Frequency of trigeminal nerve injuries following third molar removal. J Oral Maxillofac Surg. 2005; 63:732-735.

3. Hillerup S, Stoltze K. Lingual nerve injury in third molar surgery 1. Observations on recovery of sensation with spontaneous healing. Int J Oral Maxillofac Surg. 2007;36:884-889.

4. Tay ABG, Zuniga JR. Clinical Characteristics of Trigeminal Nerve Injury Referrals to a University-Based Specialist Center, Int J Oral Maxillofac Surg. 2007;36:922-927.

5. Zuniga JR, Meyer RA, Gregg JM, Miloro MD, Davis LA. The accuracy of clinical neurosensory testing for nerve injury diagnosis. J Oral Maxillofac Surg. 1998;56:2-8.

6. Zuniga JR, Essick G. A contemporary approach to the clinical evaluation of trigeminal nerve injuries. OMFS Clin North Amer. 1992; 4:353-367.

7. Cox, B, Zuniga JR, Panchal N, Cheng J, Chabbra A. Magnetic Resonance Neurography in the Management of Peripheral Trigeminal Neuropathy: experience in a tertiary care centre. Europ Radiol. 2016; DOI 10.1007/s00330-015-4182-5.

8. Zuniga JR, Chen N, Phillips CL. Chemosensory and somatosensory regeneration of the lingual epithelium following lingual nerve repair in humans. J Oral and Maxillofac Surg. 1997; 55:2-13.

9. Cheung LK, Leung YY, Chow LK, Wong MC, Chan EK, Fok YH. Incidence of neurosensory deficits and recovery after lower third molar surgery: A prospective clinical study of 4338 cases. Int J Oral Maxillofac Surg. 2010; 39:320-326. doi: 10.1016/j.ijom.2009.11.010.

10. Mason DA. Lingual nerve damage following lower third molar surgery. Int J Oral Maxillofac Surg. 1988; 17:290-294.

11. Blackburn CW. A method of assessment in cases of lingual nerve injury. Br J Oral Maxillofac Surg. 1990; 28: 238-245.

12. Bede SY, Ismael WK, Al-Assaf DA, Omer SS. Inferior alveolar nerve injuries associated with mandibular fractures. J Craniofac Surg. 2012; 23:1776-1778. doi: 10.1097/SCS.0b013e318266fda3.

13. Iannetti, G, Fadda TM, Riccardi E, Mitro V, Filiaci F. Our experience in complications of orthognathic surgery: A retrospective study on 3236 patients. Eur Rev Med Pharmacol Sci. 2013; 17:379-384.
14. Renton T, Devine M. Diagnosis and management of inferior alveolar nerve damage associated with dental implant surgery. Forum Implantologicum. 2013; 9:16-27.

15. Juodzbalys G, Wang HL, Sabalys G, Sidlauskas A, Galindo-Moreno P. Inferior alveolar nerve injury associated with implant surgery. Clin Oral Implants Res. 2013; 24:183-190. doi: 10.1111/j.16000501.2011.02314.

16. Renton T. Post-endodontic neuropathy of the trigeminal nerve. A literature review and recommendations. Endodontic Practice March 2016 http://www.endodonticpractice.co.uk/

17. Shueb SS, Nixdorf DR, John MT, Alonso BF, Durham J. What is the impact of acute and chronic orofacial pain on quality of life? J Dent. 2015; 43(10):1203-10. doi: 10.1016/j.jdent.2015.06.001. Epub 2015 Jun 12.

18. Renton T, Yilmaz Z. Profiling of patients presenting with posttraumatic neuropathy of the trigeminal nerve. J Orofac Pain. 2011; 25: 333-334.

19. Marchiori, EC, Barber JS, Williams WB et al. Neuropathic pain following sagittal split ramus osteotomy of the mandible: Prevalence, risk factors and clinical course. J Oral Maxillofac Surg. 2013; 71:21152121.

20. Robinson PP, Loescher AR,Yates JM, Smith KG. Current management of damage to the inferior alveolar and lingual nerves as a result of removal of third molars Brit J Oral Maxillofac Surg. 2004; 42:285292.

21. Smith JG, Elias LA, Yilmaz Z, et al. The psychosocial and affective burden of posttraumatic neuropathy following injuries to the trigeminal nerve. J Orofac Pain. 2013; 27:293-303.

22. Elias LA, Yilmaz Z, Smith JG, et al. Pain DETECT: A suitable screening tool for neuropathic pain in patients with painful post-traumatic trigeminal nerve injuries? Int J Oral Maxillofac Surg. 2014; 43:120126.

23. Zuniga JR, Yates D, Phillips CE. The Presence of Neuropathic Pain Predicts Postoperative Neuropathic Pain Following Trigeminal Nerve Repair. J Oral Maxillofac Surg. 2014; 72:2422-2427.

24. Zuniga JR, Yates D. Factors Determining Outcome After Trigeminal Nerve Surgery for Neuropathic Pain. J Oral Maxillofac Surg. 2016; 74:1323-1329.

25. Khawaja N, Renton T. Case studies on implant removal influencing the resolution of inferior alveolar nerve injury. Br Dent J. 2009; 206:365-370.

26. Seo K, Tanaka Y, Terumitsu M, et al. Efficacy of steroid treatment for sensory impairment after orthognathic surgery, J Oral Maxillofac Surg. 2004; 62:1193-1201.

27. Devor MR, Govrin-Lippman R. Corticosteroids suppress ectopic neural discharge originating in injured experimental neuromas. Pain. 1985; 22:127-135.

28. Khawaja N, Yilmaz Z, Renton T. Case studies illustrating the management of trigeminal neuropathic pain using topical $5 \%$ lidocaine plasters. Br J Pain. 2013; 7:107-113.

29. Center for clinical practice at NICE (UK). Neuropathic Pain: the pharmacological management of neuropathic pain in adults in nonspecialized settings (internet). London: National Institute for Health and Care Excellence, (UK); Nov. ,2013.

30. Renton T, Yilmaz Z. Management of iatrogenic trigeminal nerve damage: a case series and review of the literature. Int J Oral Maxillofac Surg. 2012; 41:629-637.

31. Herrero BA, Kapos FP, Nixdorf DR. Intraoral administration of botulinum toxin for trigeminal neuropathic pain. Oral Surg Oral Med Oral Pathol Oral Radiol. 2016; 121:148-153. 
32. Bates JAV, Nathan PW. Transcutaneous electrical nerve stimulation for chronic pain. Anesthesia. 1980; 35: 817-824.

33. Sung YF, Kutner MH, Cerine FC. Comparison of the effects of acupuncture and codeine on postoperative dental pain. Anesth Analg Curr Res. 1977; 56:473-481.
34. Poole TE, Holland I, Peterson LJ. Clinical Efficacy of low level laser treatment of oro-facial neurosensory deficits. J Oral Maxillofac Surg. 1993; 51 (suppl 3): 182-186.

35. Couthard P, Kushnerev E, Yates JM, et al. Interventions for iatrogenic inferior alveolar and lingual nerve injury. Cochrane Database Syst Rev. 2014; 16:CD005293. 